\title{
Action-at-a-distance electrodynamics in Quasi-steady-state cosmology
}

\author{
Kaustubh Sudhir Deshpande ${ }^{1,2}$ \\ (Communicated by Prof. J. V. Narlikar) \\ ${ }^{1}$ Indian Institute of Science Education and Research (IISER), Pashan, Pune 411 021, \\ India. \\ ${ }^{2}$ Current address: Harish-Chandra Research Institute, Chhatnag Road, Jhusi, Alla- \\ habad 211 019, India. \\ Email address: k.deshpande@alumni.iiserpune.ac.in
}

Keywords: Action-at-a-distance electrodynamics; Quasi-steady-state cosmology; Retarded and advanced interactions; Wheeler-Feynman absorber theory

\begin{abstract}
Action-at-a-distance electrodynamics - alternative approach to field theory - can be extended to cosmological models using conformal symmetry. An advantage of this is that the origin of arrow of time in electromagnetism can be attributed to the cosmological structure. Different cosmological models can be investigated, based on WheelerFeynman absorber theory, and only those models can be considered viable for our universe which have net full retarded electromagnetic interactions i.e. forward direction of time. This work evaluates quasi-steady-state model and demonstrates that it admits full retarded and not advanced solution. Thus QSSC satisfies this necessary condition for a correct cosmological model, based on action-at-a-distance formulation.
\end{abstract}

\section{Introduction}

Newton's law of gravitation and Coulomb's law for electrical charges, one of the very first laws of Theoretical Physics, assumed instantaneous action-ata-distance between particles. The gravitational and electrical effects due to masses and charges respectively, were assumed to travel at infinite speed in these laws. The experiments in electrodynamics, however, demonstrated that Coulomb's law was inadequate to explain their results. Gauss suggested actionat-a-distance propagating at a finite speed (speed of light) in his letter to Weber in 1845 [1]. However, this did not get immediately formulated. Instead, Maxwell developed classical field theory of electrodynamics which has effects propagating at the speed of light. This was also found to be consistent with special relativity 
(which discarded instantaneous action-at-a-distance of Newton's and Coulomb's laws). Maxwell field theory can be described by the following relativistically invariant action,

$$
S=-\sum_{a} \int m_{a} d s_{a}-\frac{1}{16 \pi} \int F_{i k} F^{i k} d^{4} x-\sum_{a} \int e_{a} A_{i} d x_{a}^{i}
$$

where $F_{i k}$ is the field, with infinite degrees of freedom, defined in terms of the 4-potential $\left(A_{i}\right)$ as $F_{i k}=\left(A_{k ; i}-A_{i ; k}\right)$. The particles (labelled by $\left.a, b, \ldots\right)$ do not interact directly with each other but interact through their coupling with the field (described by the third term in $S$ ).

In early 20th century, Schwarzschild [2], Tetrode [3] and Fokker [4, 5, 6] developed a relativistically invariant action-at-a-distance theory. This partially found the answer to Gauss's problem. This theory can be described by the Fokker action which is given as follows.

$$
S=-\sum_{a} \int m_{a} d s_{a}-\sum_{a<b} \int e_{a} e_{b} \delta\left(s_{A B}^{2}\right) \eta_{i k} d x_{a}^{i} d x_{b}^{k}
$$

The first term is the same inertial term as in the field theoretic action. The second term represents electromagnetic interactions between two different particles $a, b$ connected by a light cone ${ }^{1}$, thus preserving relativistic invariance. This action with the following definitions of direct particle potentials $\left(A_{i}^{(b)}\right)$ and fields $\left(F_{i k}^{(b)}\right)$,

$$
A_{i}^{(b)}(X)=e_{b} \int \delta\left(s_{X B}^{2}\right) \eta_{i k} d x_{b}^{k}, \quad F_{i k}^{(b)}=A_{k ; i}^{(b)}-A_{i ; k}^{(b)}
$$

gives exactly Maxwell-like equations for $F_{i k}^{(b)}$ and Lorentz-like equations of motion for the particles [7]. This formulation hence resembles and seems to provide an alternative to the Maxwell's field theory.

Action-at-a-distance in electrodynamics was provided a paradigm by Wheeler and Feynman in 1945 [8] through their absorber theory of radiation. This theory, formulated in static and flat universe, uses advanced absorber response from the entire universe as the origin for radiation reaction but, being time-symmetric in nature, allows for both retarded and advanced net interactions as consistent solutions. Wheeler and Feynman broke this symmetry in favour of the former by making an appeal to Statistical mechanics.

Extension of this formulation to expanding cosmological models (using conformal invariance of electromagnetism and conformal flatness of cosmological models) was done by Hogarth [9] and more generally by Hoyle-Narlikar [10]. Self-consistency of net advanced and retarded interactions in these models can be investigated by evaluating Wheeler-Feynman absorption integrals. Only those

\footnotetext{
${ }^{1}$ implied by $s_{A B}^{2}=\eta_{i j}\left(x_{a}^{i}-x_{b}^{i}\right)\left(x_{a}^{j}-x_{b}^{j}\right)$ being zero. $A, B$ are typical points on worldlines of the two particles $a, b$.
} 
models can be considered to be viable which have only net retarded interactions (as observed in nature). It was found by Hoyle-Narlikar that steady-state model satisfies this criterion while Friedman models do not [10].

Action-at-a-distance electrodynamics has to be first formulated for a generalized Riemannian space-time, before appyling it to cosmological space-times using conformal symmetry. This has been formulated by Hoyle-Narlikar [10, 11] by generalizing Fokker action to curved space-times using Green's functions for wave equation.

Here we evaluate Wheeler-Feynman (WF) absorption integrals in Quasisteady-state cosmology (QSSC) to investigate the self-consistency for net retarded and advanced electromagnetic interactions.

\section{Quasi-steady-state cosmology}

QSSC, based on the steady-state cosmology, is an alternative model to the standard cosmology. It was proposed by Hoyle, Burbidge and Narlikar in 1993 [12]. This is cosmological solution for Machian theory of gravity proposed by Hoyle and Narlikar in 1964 [13] with periodic creation of matter. The line element of QSSC can be represented as,

$$
\begin{aligned}
d s^{2} & =d \tau^{2}-S^{2}(\tau)\left[d r^{2}+r^{2}\left(d \theta^{2}+\sin ^{2} \theta d \phi^{2}\right)\right] \\
& =\Omega^{2}(t)\left[d t^{2}-d r^{2}-r^{2}\left(d \theta^{2}+\sin ^{2} \theta d \phi^{2}\right)\right]
\end{aligned}
$$

The scale factor ${ }^{2}$ of QSSC has the following form which is expanding exponentially over a large time scale $(P)$ and oscillating over a much shorter time scale $(Q)$. Typically, $Q=50$ gigayears, $P=20 Q$ [14].

$$
S(\tau)=\exp \left(\frac{\tau}{P}\right)[1+\eta \cos \theta(\tau)] \quad:-\infty \leq \tau \leq \infty
$$

The function $\theta(\tau)$ can be simplified and in an approximation,

$$
S(\tau)=\exp \left(\frac{\tau}{P}\right)\left[1+\eta \cos \left(\frac{2 \pi \tau}{Q}\right)\right]
$$

where $P>>Q, \eta=$ constant with $0<\eta<1$. See Fig. 1 .

The number density $(N)$ of particles oscillates between $N_{\min }$ and $N_{\max }$ during a cycle of time period $Q$ but the average density remains constant due to periodic creation of matter at particular time epochs [14].

With the following series of inequalities, we get the bounds on conformal factor, $\Omega(t)$.

$$
\exp \left(\frac{\tau}{P}\right)(1-\eta) \leq S(\tau) \leq \exp \left(\frac{\tau}{P}\right)(1+\eta)
$$

\footnotetext{
${ }^{2} S(\tau)=$ scale factor, $\Omega(t)=$ conformal factor
} 

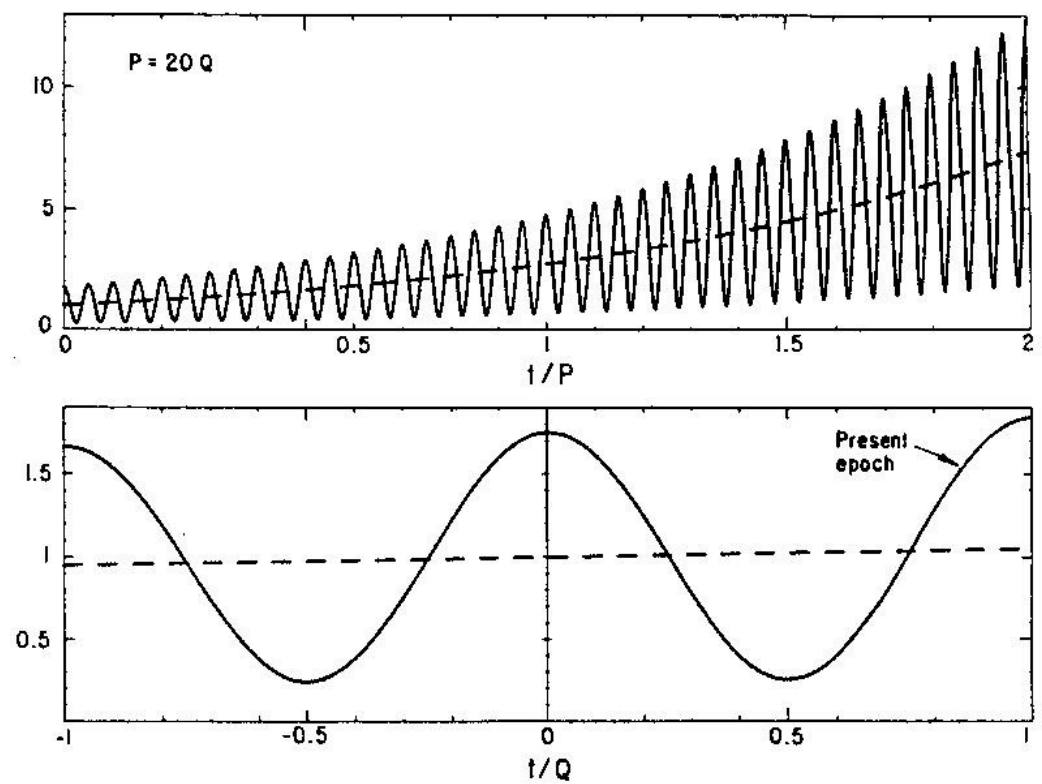

Fig. 1: Scale factor for QSSC: $S(\tau)$ expands exponentially over a time scale $P$ and oscillates over a much smaller time scale $Q$. The present epoch $\left(\tau_{0}\right)$ denotes decelerating expansion which is supported by reinterpretation of magnitude-redshift relation for supernovae in QSSC. Intergalactic dust in QSSC makes supernovae dimmer and thermalizes starlight to produce CMBR. [15, 16, 17]

$$
\begin{gathered}
\int_{\infty}^{\tau} d \tau \frac{e^{-\tau / P}}{(1-\eta)} \leq\left[t=\int_{\infty}^{\tau} \frac{d \tau}{S(\tau)}\right] \leq \int_{\infty}^{\tau} d \tau \frac{e^{-\tau / P}}{(1+\eta)} \quad:-\infty \leq t \leq 0 \\
-\frac{P}{t}\left(\frac{1-\eta}{1+\eta}\right) \leq e^{\tau / P}(1-\eta) \leq \Omega(t) \leq e^{\tau / P}(1+\eta) \leq-\frac{P}{t}\left(\frac{1+\eta}{1-\eta}\right) \\
-\frac{P}{t}\left(\frac{1-\eta}{1+\eta}\right) \leq \Omega(t) \leq-\frac{P}{t}\left(\frac{1+\eta}{1-\eta}\right)
\end{gathered}
$$

Thus (9) denotes the bounds on conformal factor $\Omega(t)$. It is important to note that the oscillatory microstructure in the scale factor $S(\tau)$ is not being ignored or approximated here. We are instead considering bounds on the functional forms, in an appropriate way. Hence the results obtained in the next section (regarding WF absorption integrals) are exact and not approximate. 


\section{Evaluation of WF absorption integrals: retarded and advanced waves}

In this section we evaluate WF absorption integrals $\left(I_{R}\right.$ and $\left.I_{A}\right)$ in QSSC, to check self-consistency of retarded $(R)$ and advanced $(A)$ solutions. We are using Hoyle-Narlikar approach [10] for this evaluation, with radiation reaction provided by absorber response from the entire universe. The conditions required for this are given by the divergences of the following integrals ${ }^{3}$.

$I_{R}=\int_{\text {future }} k(r) d r \rightarrow-\infty, I_{A}=\int_{\text {past }} k(r) d r \rightarrow+\infty \quad ; k=$ absorption coefficient

Cosmological shift in the frequency of wave ${ }^{4}$ is given by,

$$
\begin{gathered}
\omega \propto S^{-1} \propto \Omega^{-1} \\
\omega_{R}=\omega_{0}\left(\Omega^{-1}\left(t_{0}+r\right)\right), \quad \omega_{A}=\omega_{0}\left(\Omega^{-1}\left(t_{0}-r\right)\right)
\end{gathered}
$$

Using (9), $\omega_{R}$ and $\omega_{A}$ satisfy the following inequalities.

$$
\begin{aligned}
& -\omega_{0} \frac{\left(t_{0}+r\right)}{P}\left(\frac{1-\eta}{1+\eta}\right) \leq \omega_{R} \leq-\omega_{0} \frac{\left(t_{0}+r\right)}{P}\left(\frac{1+\eta}{1-\eta}\right) \\
& -\omega_{0} \frac{\left(t_{0}-r\right)}{P}\left(\frac{1-\eta}{1+\eta}\right) \leq \omega_{A} \leq-\omega_{0} \frac{\left(t_{0}-r\right)}{P}\left(\frac{1+\eta}{1-\eta}\right)
\end{aligned}
$$

The refractive index satisfies the following expressions in the respective lim$\operatorname{its}^{5}[10]$.

$$
1-(n-i k)^{2}= \begin{cases}\frac{4 \pi N e^{2}}{m \omega^{2}}\left[1-\frac{2 i e^{2}}{3 m} \omega+O\left(\omega^{2}\right)\right] & ; \omega \rightarrow 0 \\ \frac{4 \pi N e^{2}}{m \omega^{2}}\left[1+O\left(\frac{1}{\omega}\right)\right] & ; \omega \rightarrow \infty\end{cases}
$$

Hence absorption coefficient $(k)$ satisfies,

$$
k_{R} \sim-\frac{\sqrt{N}}{\omega}, \quad k_{A} \sim \frac{N}{\omega^{3}}
$$

Using (13) for retarded solution and the fact that $N_{\min } \leq N \leq N_{\max }$ for QSSC,

$$
\frac{\sqrt{N_{\max }}}{\omega_{0}} P\left(\frac{1+\eta}{1-\eta}\right) \frac{1}{\left(t_{0}+r\right)} \leq\left[k_{R} \sim-\frac{\sqrt{N}}{\omega}\right] \leq \frac{\sqrt{N_{\min }}}{\omega_{0}} P\left(\frac{1-\eta}{1+\eta}\right) \frac{1}{\left(t_{0}+r\right)}
$$

\footnotetext{
${ }^{3}$ for spatially flat universe

${ }^{4}$ The range and constraints on coordinates - for retarded wave: $r=t-t_{0}, t: t_{0} \rightarrow 0, r$ : $0 \rightarrow-t_{0}$; for advanced wave: $r=t_{0}-t, t: t_{0} \rightarrow-\infty, r: 0 \rightarrow \infty$.

${ }^{5} \omega \rightarrow 0$ and $\omega \rightarrow \infty$ limits correspond to future and past infinities respectively.
} 


$$
\frac{C_{2}}{t_{0}+r} \leq k_{R} \leq \frac{C_{1}}{t_{0}+r}
$$

The future absorption integral $\left(I_{R}\right)$ thus satisfies ${ }^{6}$,

$$
C_{2} \int_{0}^{-t_{0}} \frac{d r}{t_{0}+r} \leq\left[I_{R}=\int_{\text {future }} k_{R}(r) d r\right] \leq C_{1} \int_{0}^{-t_{0}} \frac{d r}{t_{0}+r}
$$

As integrals on either side of $I_{R}$ in the above inequality diverge to $-\infty, I_{R}$ also diverges to $-\infty$. Thus QSSC satisfies the condition for $I_{R}$ (see (10)) and admits self-consistent retarded solution.

Similarly using (14) for advanced solution,

$$
\begin{gathered}
-\frac{N_{\min }}{\omega_{0}^{3}} P^{3}\left(\frac{1-\eta}{1+\eta}\right)^{3} \frac{1}{\left(t_{0}-r\right)^{3}} \leq\left[k_{A} \sim \frac{N}{\omega^{3}}\right] \leq-\frac{N_{\max }}{\omega_{0}^{3}} P^{3}\left(\frac{1+\eta}{1-\eta}\right)^{3} \frac{1}{\left(t_{0}-r\right)^{3}} \\
\frac{D_{2}}{\left(r-t_{0}\right)^{3}} \leq k_{A} \leq \frac{D_{1}}{\left(r-t_{0}\right)^{3}}
\end{gathered}
$$

The past absorption integral $\left(I_{A}\right)$ satisfies $^{7}$,

$$
\begin{gathered}
D_{2} \int_{0}^{\infty} \frac{d r}{\left(r-t_{0}\right)^{3}} \leq\left[I_{A}=\int_{\text {past }} k_{A}(r) d r\right] \leq D_{1} \int_{0}^{\infty} \frac{d r}{\left(r-t_{0}\right)^{3}} \\
\Rightarrow \frac{D_{2}}{2 t_{0}^{2}} \leq I_{A} \leq \frac{D_{1}}{2 t_{0}^{2}}
\end{gathered}
$$

This shows that $I_{A}$ takes a finite value and hence QSSC does not admit self-consistent advanced solution.

It is important to note that the absorption property derived here (for both future and past absorption) is a robust result. It is independent of where the observer is located. (This is also evident from the fact that nowhere in the calculation the value $\tau_{0}$, or local qualitative behaviour related to its position, is required). Hence the same result applies to observers located at epochs corresponding to deep crest or trough of the oscillation. This is because the underlying theory being global (and not local) in nature, one has to consider absorber response from the entire universe. This amounts to evaluating WF absorption integrals $I_{R}$ and $I_{A}$ till future and past infinities, respectively. Thus local variations or position of the observer do not affect the results in any significant way.

\footnotetext{
${ }^{6} C_{1}$ and $C_{2}$ are positive, finite constants. $C_{1}=\frac{\sqrt{N_{\min }}}{\omega_{0}} P\left(\frac{1-\eta}{1+\eta}\right), C_{2}=\frac{\sqrt{N_{\max }}}{\omega_{0}} P\left(\frac{1+\eta}{1-\eta}\right)$

${ }^{7} D_{1}$ and $D_{2}$ are positive, finite constants. $D_{1}=\frac{N_{\max }}{\omega_{0}^{3}} P^{3}\left(\frac{1+\eta}{1-\eta}\right)^{3}, D_{2}=\frac{N_{\min }}{\omega_{0}^{3}} P^{3}\left(\frac{1-\eta}{1+\eta}\right)^{3}$
} 


\section{Additional supplementary calculation of WF integrals}

In order to support the note made in the last paragraph of previous section, we present an additional analysis in this section to demonstrate that the selfconsistency conditions in QSSC are independent of $\frac{P}{Q}$ or local position of the observer (i.e. in crest or trough of the oscillation).

Null, radial light ray in QSSC satisfies the following.

$$
\frac{d r}{d \tau}=\frac{1}{S(\tau)}, \quad S(\tau)=\exp \left(\frac{\tau}{P}\right)\left[1+\eta \cos \left(\frac{2 \pi \tau}{Q}\right)\right]
$$

Using (11), we have $\omega(\tau)=\frac{\omega_{0}}{S(\tau)}$.

Now, using the dependence of $k_{R}$ and $k_{A}$ on $\omega$ as given in (16), we obtain the following results for $I_{R}$ and $I_{A}$.

$$
\begin{gathered}
I_{R}=\int_{\text {future }} k_{R} d r=\int_{\tau_{0}}^{\infty} k_{R} \frac{d r}{d \tau} d \tau \sim-\int_{\tau_{0}}^{\infty} \sqrt{N} d \tau \\
-\sqrt{N_{\max }} \int_{\tau_{0}}^{\infty} d \tau \leq\left[I_{R} \sim-\int_{\tau_{0}}^{\infty} \sqrt{N} d \tau\right] \leq-\sqrt{N_{\min }} \int_{\tau_{0}}^{\infty} d \tau
\end{gathered}
$$

Hence it can be seen that, $I_{R} \rightarrow-\infty$, completely independent of the value $\frac{P}{Q}$ or $\tau_{0}^{8}$.

$$
\begin{gathered}
I_{A}=\int_{\text {past }} k_{A} d r=\int_{\tau_{0}}^{-\infty} k_{A} \frac{d r}{d \tau} d \tau \sim \int_{\tau_{0}}^{-\infty} N S^{2}(\tau) d \tau \\
N_{\min } \int_{\tau_{0}}^{-\infty} S^{2}(\tau) d \tau \leq\left[I_{A} \sim \int_{\tau_{0}}^{-\infty} N S^{2}(\tau) d \tau\right] \leq N_{\max } \int_{\tau_{0}}^{-\infty} S^{2}(\tau) d \tau \\
\Lambda \equiv \int_{\tau_{0}}^{-\infty} S^{2}(\tau) d \tau=\frac{P}{4} e^{2 \tau_{0} / P}\left[\frac{1}{\left(4 \pi^{2} P^{2}+Q^{2}\right)}\left\{2 \pi \eta^{2} P Q \sin \left(\frac{4 \pi \tau_{0}}{Q}\right)+\eta^{2} Q^{2} \cos \left(\frac{4 \pi \tau_{0}}{Q}\right)\right\}\right. \\
\left.+\frac{1}{\left(\pi^{2} P^{2}+Q^{2}\right)}\left\{4 \pi \eta P Q \sin \left(\frac{2 \pi \tau_{0}}{Q}\right)+4 \eta Q^{2} \cos \left(\frac{2 \pi \tau_{0}}{Q}\right)\right\}+\eta^{2}+2\right]
\end{gathered}
$$

Considering $P=n \frac{Q}{2 \pi}$,

$$
\begin{aligned}
\Lambda & =\frac{Q}{2 \pi} n e^{4 \pi \tau_{0} / n Q}\left[\frac{\eta^{2}}{1+n^{2}}\left\{n \sin \left(\frac{4 \pi \tau_{0}}{Q}\right)+\cos \left(\frac{4 \pi \tau_{0}}{Q}\right)\right\}\right. \\
& \left.+\frac{8 \eta}{4+n^{2}}\left\{n \sin \left(\frac{2 \pi \tau_{0}}{Q}\right)+2 \cos \left(\frac{2 \pi \tau_{0}}{Q}\right)\right\}+\eta^{2}+2\right]
\end{aligned}
$$

In the case of pure oscillations $\left(n \rightarrow \infty\right.$ limit), i.e. with $\exp \left(\frac{\tau}{P}\right)$ term absent in $S(\tau), \Lambda \rightarrow \infty$ and hence $I_{A} \rightarrow \infty$. Thus since both $I_{R}$ and $I_{A}$ diverge in

\footnotetext{
${ }^{8} \tau_{0}$ is the present epoch i.e. epoch of the position of the observer.
} 
this case, both future and past absorbers are perfect. This model of purely oscillatory universe thus has ambiguous outcome for causality and an external criterion (e.g. statistical mechanics, as used in [8] for flat space-time) would be required to decide the direction of time.

In the pure expansion case $(Q \rightarrow \infty$ limit), i.e. with the oscillatory term absent in $S(\tau), \Lambda=$ finite and hence $I_{A}=$ finite. This limit corresponds to Steady-state cosmological model and admits self-consistent retarded but not advanced solution. This result agrees with that demonstrated in [10, 11].

In QSSC model, however, the time scales $P$ and $Q$ assume positive, finite and non-zero values. Hence the value of $n$ is large ${ }^{9}$ but finite. For any finite non-zero value of $n, \Lambda$ and hence $I_{A}$ converge to finite values. Hence QSSC satisfies self-consistency condition for retarded but not advanced solution.

It can also be noted that all the above results are unchanged for any value of $\frac{\tau_{0}}{Q}$, i.e. position of the observer in oscillation cycle. Hence the self-consistency conditions are the same for observers located in crests or troughs of the oscillation.

\section{Conclusion}

The above calculations show that in QSSC, $I_{R}=\int_{\text {future }} k(r) d r \rightarrow-\infty$ and $I_{A}=\int_{\text {past }} k(r) d r \rightarrow$ finite value. This thus concludes, in a self consistent way, that QSSC assumes only net full retarded electromagnetic interactions. This causality is applicable at all time epochs, as illustrated in the previous section. QSSC is thus a viable cosmological model according to action-at-a-distance formulation. Though this cannot be the sufficient criterion to decide for the correct cosmological model, it certainly is a necessary criterion, according to this approach.

Also, this has interesting implications on the origin of arrow of time. The choice of the direction of time is ad hoc in field theory i.e. the retarded solution is chosen arbitrarily over the advanced one. However in action-at-a-distance formulation, origin of time asymmetry can be attributed to the large scale structure of the universe. The universe has such a cosmological stucture that it provides the correct absorber response to produce net retarded interactions, thus fixing the arrow of time. Quasi-steady-state model is a suitable candidate for such a cosmological structure, as shown in the present work.

Acknowledgements I would like to express deep gratitude towards Prof. J. $\mathrm{V}$ Narlikar for suggesting this problem to work on and his constant guidance through discussions. I am grateful towards IUCAA Pune and IISER Pune for providing access to the facilities at these institutes. I would also like to thank the (anonymous) journal referee for providing useful comments and suggestions.

\footnotetext{
${ }^{9}$ as $P>>Q$
} 


\section{References}

[1] C F Gauss, Werke 5, 629 (1867)

[2] K Schwarzschild, Gottinger Nachrichten, 128, 132 (1903)

[3] H Tetrode, Z. Phys. 10, 317 (1922)

[4] A D Fokker, Z. Phys. 58, 386 (1929)

[5] A D Fokker, Physica 9, 33 (1929)

[6] A D Fokker, Physica 12, 145 (1932)

[7] F Hoyle and J V Narlikar, Rev. Mod. Phys., Vol. 67, No. 1, 113 (1995)

[8] J A Wheeler and R P Feynman, Rev. Mod. Phy., 17, 157 (1945)

[9] J E Hogarth, Proc. Roy. Soc. A, 267, 365 (1962)

[10] F Hoyle and J V Narlikar, Proc. Roy. Soc. A, 277, 1 (1964)

[11] F Hoyle and J V Narlikar, Action at a distance in Physics and Cosmology (W H Freeman and Company, San Francisco, 1974), Chap. 5, p. 85

[12] F Hoyle, G Burbidge, J V Narlikar, The Astrophy. J., 410, 437 (1993)

[13] F Hoyle, J V Narlikar, Proc. R. Soc. Lond. A, 282, 191 (1964)

[14] J V Narlikar, An Introduction to Cosmology, 3rd edition (Cambridge University Press, 2010), Chap. 9, p. 347

[15] H Kragh, arXiv:1201.3449v1 [physics.hist-ph] (2012)

[16] J V Narlikar, R G Vishwakarma and G Burbidge, Pub. of the Astron. Soc. of the Pac., 114, 800, 1092 (2002)

[17] F Hoyle, G Burbidge and J V Narlikar, A different approach to cosmology (Cambridge University Press, 2000), Chap. 16, p. 197 